\title{
To Asymptotic of the Solution of the Heat Conduction Problem with Double Nonlinearity with Absorption at a Critical Parameter
}

\author{
Aripov M. , Mukimov A., Mirzayev B. \\ National University of Uzbekistan Named after M. Ulugbek, Uzbekistan
}

Received September 17, 2019; Revised October 30, 2019; Accepted November 12, 2019

Copyright $\bigcirc 2019$ by authors, all rights reserved. Authors agree that this article remains permanently open access under the terms of the Creative Commons Attribution License 4.0 International License

\begin{abstract}
We study the asymptotic behavior (for $t \rightarrow \infty$ ) of solutions of the Cauchy problem for a nonlinear parabolic equation with a double nonlinearity, describing the diffusion of heat with nonlinear heat absorption at the critical value of the parameter $\beta$. For numerical computations as an initial approximation we used founded the long time asymptotic of the solution. Numerical experiments and visualization were carried for one and two dimensional case.
\end{abstract}

Keywords Heat Conduction Problem, Critical Value of Parameter, Absorption, Maximum Principle, Numerical Computation, Visualization

\section{Introduction}

As is well known for the numerical computation of a nonlinear problem, the choice of the initial approximation is essential, which preserves the properties of the final speed of propagation, spatial localization, bounded and blow-up solutions, which guarantees convergence with a given accuracy to the solution of the problem with minimum number of iterations.

It is very important to establish the values of numerical parameters at which the nature of the asymptotic behavior of the solution will change. Such values of numeric parameters are called critical or critical values of the Fujita type. He first established this for the semi-linear heat equation [1]. At the critical parameters we can observe new effects such as infinite energy, localization and others.

In the domain $Q=\left\{(t, x): t>0, x \in R^{N}\right\}$ the following Cauchy problem

$$
\frac{\partial u}{\partial t}=\nabla\left(u^{m-1}\left|\nabla u^{k}\right|^{p-2} \nabla u\right)-u^{\beta_{*}}
$$

$$
u(0, x)=u_{0}(x), x \in R^{N}
$$

$\mathrm{t}$ and $\mathrm{x}$ are, respectively, the temporal and spatial coordinates where $m \geq 1, k \geq 1, p \geq 2$ given numerical parameters, characterizing the nonlinear medium $\nabla(\cdot)=\underset{x}{\operatorname{grad}(\cdot)}, \quad \beta_{*}=k(p-2)+m+\frac{p}{N}\left(\beta_{*}\right.$-critical value, $\mathrm{N}$-size of dimension). In case $\mathrm{k}=1$, critical value of parameter $\beta_{*}$ was found in [2].

Problem (1.1) - (1.2) is the basis for modeling various processes of nonlinear heat diffusion, magnetic hydrodynamics, gas and liquid filtration, oil and gas, in the theory of non-Newtonian fluids, etc.

Under some suitable assumptions, the existence, uniqueness and regularity of a weak solution to the Cauchy problem (1.1) - (1.2) and their variants have been extensively investigated by many authors (see [3-5] and the references therein).

If the initial value $u(x, 0)=u_{0}(x)$ is respectively smooth, there are many papers on the solvability of the Cauchy problem (1.1)-(1.2), we can refer to Wu-Zhao [6], Gmira [7], Yang-Zhao [8], Zhao [9-11], Zhao-Yuan [12], Dibenedetto-Friedman [13], LiHia [14], Dibenedetto-Herrero [15], Beniland-Crandal Pierre [16], Zhao-Xu [17], Fan [18] and links to them for details.

A lot of works studied properties of solutions of problem with critical value of parameter $\beta$ and were established asymptotic behavior for $t \rightarrow \infty$ (see [1], [2], [19]-[27]). The long time asymptotic of solutions has been established for the critical value of parameter $\beta$ for problem (1.1)-(1.2) in case $\mathrm{k}=1, \mathrm{~m}=1, \beta_{*}=p-1+p / N$ in [19], case $\mathrm{p}=2, \beta_{*}=m+2 / N$ in [20].

In [21] authors were established the long time asymptotic of solutions for the critical value of parameter 
$\beta=1+\frac{2}{N}$ for problem (1.1) - (1.2) in case $\mathrm{m}=1, \mathrm{p}=2$.

They considered following semi-linear parabolic equation

$$
\begin{gathered}
u_{t}-\Delta u+u^{\beta}=0, t>0 \\
u(0, x)=u_{0}(x) \geq 0 \\
\Delta=\sum_{i=1}^{N} \partial^{2} / \partial x_{i}^{2}, \beta=1+\frac{2}{N}
\end{gathered}
$$

The solution of problem (3) - (4) is "infinity" energy. The initial data is

$$
\begin{gathered}
u_{0}(x)=o\left\{\exp \left(-\gamma|x|^{2}\right)\right\}, x \rightarrow+\infty \\
\gamma>0
\end{gathered}
$$

They proved that for problem (3) - (4) the long time asymptotic of the solutions is the following approximate self-similar solution

$$
u(t, x)=[(T+t) \ln (T+t)]^{-\frac{N}{2}} g_{*}\left(\frac{x}{(T+t)^{\frac{1}{2}}}\right)
$$

For $g_{*}$ function upper and lower bounds were obtained

$$
A \exp \left(-\frac{|\xi|^{2}}{4}\right) \leq g_{*}(\xi) \leq H \exp \left(-\frac{|\xi|^{2}}{4}\right)|\xi|^{2}=\frac{|x|^{2}}{T+t}
$$

where $\mathrm{A}, \mathrm{H}$-constants.

For $\beta \neq 1+\frac{2}{N}$, the approximate self-similar differs from (5), which means that for critical values the asymptotic of the solutions will change for $t \rightarrow \infty$.

In [22] was considered following nonlinear heat equation with absorption

$$
\begin{gathered}
u_{t}=\Delta\left(\mathrm{u}^{\sigma+1}\right)-\mathrm{u}^{\beta} \text { in area } Q=R^{N} \times(0, \infty) \\
u(x, 0)=u_{0}(x) \text { for } x \in R^{N}
\end{gathered}
$$

Authors established the long time asymptotic of the solution for the critical exponent $\beta=\beta_{*}=\sigma+1+2 / N$.

The following asymptotic

$$
\begin{gathered}
\mathrm{u}(\mathrm{x}, \mathrm{t})=((T+t) \ln (\mathrm{T}+\mathrm{t}))^{-k} \mathrm{~F}(\xi ; \mathrm{a}) \\
\xi=x(\mathrm{~T}+\mathrm{t})^{-k / N} \ln (\mathrm{T}+\mathrm{t})^{k \sigma / 2} \\
k=N /(\mathrm{N} \sigma+2), \mathrm{F}(\xi ; \mathrm{a})=\mathrm{C}_{0}\left(\mathrm{a}^{2}-|\xi|^{2}\right)_{+}^{1 / \sigma}, \\
\mathrm{C}_{0}=[k \sigma / 2 N(\sigma+1)]^{1 / \sigma}, T>1
\end{gathered}
$$

where the value of the numerical parameter a is determined from the law of energy conservation

$$
\begin{gathered}
\int w_{M}(\mathrm{x}, \mathrm{t}) \mathrm{dx}=\int F(\xi ; \mathrm{a}) \mathrm{d} \xi=M \\
M=C_{1} a^{N / k \sigma}
\end{gathered}
$$

where $C_{1}=\pi^{N / 2} C_{0} B(\mathrm{~N} / 2,1+1 / \sigma) / \Gamma(\mathrm{N} / 2)$

$\mathrm{B}$ and $\Gamma$ is beta and gamma of Euler function.

They proved that solution (8) is the long time asymptotic of the solution to problem (6) - (7) by constructing lower and upper solutions. The following lower and upper solution with variable a

$$
\begin{gathered}
((T+t) \log (\mathrm{T}+\mathrm{t}))^{-k} F\left(\xi ; \mathrm{a}_{-}\right) \leq \mathrm{u}(\mathrm{x}, \mathrm{t}) \leq \\
\leq((T+t) \log (\mathrm{T}+\mathrm{t}))^{-k} F\left(\xi ; \mathrm{a}_{-}\right) \\
0<a_{-}<a_{+}
\end{gathered}
$$

In [23] authors gave bounds for the blow-up rate of solution the following nonlinear parabolic equation

$$
\begin{gathered}
u_{t}-\operatorname{div}\left(u^{m-1}|D u|^{\lambda-1} D u\right)=f(x) u^{p} \text { in } \\
Q_{T}=\Omega x(0, T), 0<T<\infty
\end{gathered}
$$

where $f(x)$ is a radial function and for simplicity it taken like this $f(x)=|x|^{-\alpha},-\infty<\alpha<\min (N, \lambda+1)$ Here are some of them

$$
u(x, t) \leq \gamma(T-t)^{-B}
$$

Where $\quad \gamma>0 \quad, \quad|x|<(T-t)^{1 / H} / 2 \quad$, $0<m+\lambda-1<p<m+\lambda-1+(\lambda+1-\alpha) / N$ $H=\frac{(p-1)(\lambda+1)-\alpha(m+\lambda-2)}{p-m-\lambda+1}$, $B=\frac{\lambda+1-\alpha}{(p-1)(\lambda+1)-\alpha(m+\lambda-2)}$

( $\mathrm{H}$ gives the correct space-time scaling near the blow-up time, B gives the blow-up rate).

In [24] established conditions of norm of solution with critical exponent $q^{*}=\frac{K+N}{N v+1}$ for following Cauchy problem

$$
\begin{gathered}
u_{t}-\operatorname{div}\left(u^{m-1}|D u|^{\lambda-1} D u\right)=-\varepsilon\left|D u^{v}\right|^{q_{*}}+\delta u^{p} \text { in } \\
R^{N} \times(0, T) \\
u(x, 0)=u_{0}(x) \geq 0
\end{gathered}
$$

Here are some of them

$$
\begin{aligned}
& \text { if } q<q^{*} \text { then }\|u(t)\| \leq \gamma t^{-A} \\
& \text { if } q=q^{*} \text { then }\|u(t)\| \leq \gamma[\ln t]^{-\frac{1}{v q-1}}
\end{aligned}
$$


here $A=\frac{q^{*}-q}{H}(N v+1), H=(\lambda+1)(v q-1)-q(m+\lambda-2)$,

$$
\begin{aligned}
& K=N(m+\lambda-2)+\lambda+1, \\
& \delta=0, \varepsilon=1, m+\lambda-2>0 .
\end{aligned}
$$

In [25] the long time asymptotic of the solution were established for the following problem with critical parameter $p_{c}=1+2 m / N$

$$
\begin{gathered}
u_{t}=-(-\Delta)^{m}-|u|^{p-1} u \text { in area } R^{N} \times R_{+} \\
u(x, 0)=u_{0}(x)
\end{gathered}
$$

Following asymptotic

$$
u(x, t)= \pm C_{0} t^{-N / 2 m}(\ln t)^{-N /(2 m+Q)}\left[f\left(\frac{x}{t^{1 / 2 m}}\right)+o(1)\right]
$$

where $f$ is the rescaled kernel of the fundamental solution of the linear parabolic operator.

Mu et al. [26] studied the secondary critical exponent for the following p-Laplacian equation with slow decay initial values:

$$
\begin{gathered}
u_{t}=\operatorname{div}\left(|\nabla u|^{p-2} \nabla u\right)+u^{q},(x, t) \in R^{N} \times(0, T) \\
u(x, 0)=u_{0}(x), x \in R^{N}
\end{gathered}
$$

where $\mathrm{p}>2, \mathrm{q}>1$, and showed that, for $q>q_{c}^{*}=p-1+(p / N)$, there exists a secondary critical exponent $a_{c}^{*}=(p /(q+1-p))$ such that the solution $u(x, t)$ of (1.9)-(1.10) blows up in finite time for the initial data $u_{0}(x)$ which behaves like $|x|^{-\alpha}$ at $x=\infty$, if $a \in\left(0, a_{c}^{*}\right)$, and there exists a global solution for the initial data $u_{0}(x)$, which behaves like $|x|^{-\alpha}$ at $x=\infty$, if $a \in\left(a_{c}^{*}, N\right)$.

J.-S. Guo and Y. Y. Guo [27] obtained the secondary critical exponent for the following porous medium type equation in high dimensions:

$$
\begin{gathered}
u_{t}=\Delta u^{m}+u^{p},(x, t) \in R^{N} \times(0, T) \\
u(x, 0)=u_{0}(x), x \in R^{N}
\end{gathered}
$$

where $\mathrm{p}>1, \mathrm{~m}>1$ or $\max \{0,1-(2 / \mathrm{N})\}<\mathrm{m}<1, u_{0}(x)$ is nonnegative bounded and continuous, and proved that for $p>p_{m}^{*}=m+(2 / N)$, there exists a secondary critical exponent $a^{*}=2 /(p-m)$ such that the solution $u(x, t)$ of (1.11)- (1.12) blows up in finite time for the initial data $u_{0}(x)$.

The authors of [28] considered the fast diffusion equation for large $t$, when the degree of absorption is critical for the following equation

$$
\begin{gathered}
\partial_{t} u-\Delta u^{m}+u^{q}=0 \text { in area }(0, \infty) \mathrm{xR}^{N} \\
u(0, \mathrm{x})=\mathrm{u}_{0}(\mathrm{x}), \mathrm{x} \in \mathrm{R}^{N} \\
q=q_{*}:=m+\frac{2}{N}
\end{gathered}
$$

They established a clear lower bound, which eliminates convergence to zero.

In this paper, we study the asymptotic behavior (for $t \rightarrow \infty$ ) of solutions of the Cauchy problem (1)-(2) for a nonlinear parabolic equation with a double nonlinearity, describing the diffusion of heat with nonlinear heat absorption at the critical value of the parameter $\beta$.

\section{Asymptotic of the Solution}

Based on the method of standard equation [2], the solution to the problem (1)-(2) will be find in the following form

$$
u(t, x)=\bar{u}(t) \omega(\tau(t), x), \bar{u}(t)=(t \ln t)^{-\frac{1}{\beta_{s}-1}}
$$

Put (13) in (1) and select $\tau(t)$

$$
\tau(t)=\int(t \ln t)^{-\frac{m+k(p-2)-1}{\beta_{*}-1}} d t
$$

For $\omega(\tau(t), x)$ we get following equation

$$
\begin{aligned}
& \frac{\partial \omega}{\partial \tau}=\nabla\left(\omega^{m-1}\left|\nabla \omega^{k}\right|^{p-2} \nabla \omega\right)-\bar{u}^{\beta-(m+k(p-2))} \omega^{\beta}- \\
& -\frac{d \bar{u}}{d t} \bar{u}^{-(m+k(p-2))} \omega
\end{aligned}
$$

We note that for large $t$ by the Hardy theorem on the behavior of the integral [2], the function $\tau(t)$ has the following asymptotic

$$
\tau(t) \sim t^{\alpha_{1}} \ln ^{\alpha_{2}} t, t \sim \infty
$$

Where

$$
\begin{aligned}
& \alpha_{1}=\frac{k(p-2)+m-1}{(p+(k(p-2)+m) N) p}, \\
& \alpha_{2}=-\frac{k(p-2)+m-1}{(p+(k(p-2)+m) N) p}+1
\end{aligned}
$$

Now put $\omega(\tau, x)=f(\xi), \quad \xi=x[\tau(t)]^{-1 / p}$, in (14) we get an approximately self-similar equation: 


$$
\begin{aligned}
& \xi^{1-N} \frac{d}{d \xi}\left(\xi^{N-1} f^{m-1}\left|\frac{d f^{k}}{d \xi}\right|^{p-2} \frac{d f}{d \xi}\right)+\frac{\xi}{p} \frac{d f}{d \xi}- \\
& -f \tau\left(\bar{u}^{-\beta-(m+k(p-2))} f^{\beta-1}-\frac{d \bar{u}}{d t} \bar{u}^{-(m+k(p-2))}\right)=0
\end{aligned}
$$

We take as a generalized solution of the equation (15) the following function

$$
\begin{gathered}
\bar{f}(\xi)=\left(a-b \xi^{\frac{p}{p-1}}\right)_{+}^{\frac{p-1}{m+k(p-2)-1}} \\
b=(m+k(p-2)-1) /\left(k^{p-2} p^{\frac{p}{p-1}}\right), m+k(p-2)-1>0
\end{gathered}
$$

After putting (16) into (15) a self-similar equation transforms to

$$
L(f)=-\frac{N}{p} \bar{f}-\bar{f} \tau u^{-\frac{p}{N}}\left(\bar{f}^{\beta_{*}-1}+\frac{1}{\beta-1}(\ln t+1)\right)
$$

\section{Theorem 1}

$$
\text { Let }(z-1)\left(\beta_{*}-1\right)-(p+z N) p<0 \text { and }
$$

$\left(\beta_{*}-1\right)((p+z(N-1)) p-z+1)-p(p+z N)<$

Where

$$
z=k(p-2)+m, \beta_{*} \text { mentioned number }
$$

Then for $t \rightarrow+\infty$ solution of problem (1) - (2) bounded above by function $u_{+}(t, x)$

$$
\xi, b, \bar{u}(t) \text { defined above }
$$

Proof

Show that $u_{+}(t, x)$ is the upper solution of problem (1) - (2). After putting $u_{+}(t, x)$ in (17) we get the following estimates

$$
L(\bar{f})=\bar{f}\left(\frac{1}{\beta-1} \bar{u}^{-\frac{p}{N}}(\ln t+1) \tau-\frac{N}{p}-\tau \bar{u}^{-\frac{p}{N}} \bar{f}^{\beta_{x}-1}\right) \leq 0
$$

To satisfy these estimates, the following conditions are sufficient

$$
\begin{aligned}
& \frac{1}{\beta_{*}-1} t^{\frac{(z-1)\left(\beta_{*}-1\right)-(p+z N) p}{\left(\beta_{*}-1\right)(p+z N) p}} \ln \frac{\left(\beta_{*}-1\right)((p+z(N-1)) p-z+1)-p(p+z N)}{\left(\beta_{*}-1\right)(p+z N) p} t(\ln t+1)- \\
& -\frac{N}{P} \leq 0
\end{aligned}
$$

These conditions valid due to conditions of the theorem 1.

Theorem 2.

$$
\text { Let } \quad a \leq\left[\frac{1}{\beta_{*}-1}\right]^{\frac{m+k(p-2)-1}{(p-1)\left(\beta_{*}-1\right)}}
$$

$$
\begin{aligned}
& (z-1)\left(\beta_{*}-1\right)-(p+z N) p>0 \text { and } \\
& \left(\beta_{*}-1\right)((p+z(N-1)) p-z+1)-p(p+z N)>0
\end{aligned}
$$

$z=k(p-2)+m, \beta_{*}$ defined above

Then for $t \rightarrow+\infty$ solution of problem (1) -

bounded below by function $u_{-}(t, x)$

$$
\begin{aligned}
& u(t, x) \geq u_{-}(t, x)=\bar{u}(t) \bar{f}\left(\xi\left(1+\ln ^{-1} t\right)\right), \bar{f}\left(\xi\left(1+\ln ^{-1} t\right)\right)= \\
& =\left(a-b \xi^{\frac{p}{p-1}}\left(1+\frac{1}{\ln t}\right)\right)_{+}^{\frac{p-1}{m+k(p-2)-1}}
\end{aligned}
$$

Proof

Show that $u_{-}(t, x)$ is the lower solution of problem (1) - (2). After putting $u_{-}(t, x)$ in (17), we get the following estimates

$$
\bar{f}\left(\xi\left(1+\ln ^{-1} t\right)\right)\left(\tau(t) u^{-\frac{p}{N}}\left[\frac{1}{\beta-1}(\ln t+1)-\bar{f}\left(\xi\left(1+\ln ^{-1} t\right)\right)^{\beta-1}\right]-\frac{N}{p}\right) \geq 0
$$

To satisfy these estimates, the following conditions are sufficient

$$
\frac{1}{\beta_{*}-1}+\frac{\ln t}{\beta_{*}-1}-\frac{N}{p \tau \overline{u^{N}}}-\bar{f}\left(\xi\left(1+\ln ^{-1} t\right)\right)^{\beta-1} \geq 0
$$

These conditions are true due to conditions of the theorem 2.

From the last two theorems it follows that for all large $t$ the self-similar solution $\bar{u}(t) \bar{f}(\xi)$ is bounded above and below

$$
u_{+}(t, x)=\bar{u}(t) \bar{f}(\xi) \geq u(t, x) \geq u_{-}(t, x)=\bar{u}(t) \bar{f}\left(\xi\left(1+\ln ^{-1} t\right)\right)
$$

For $t \rightarrow+\infty$ we get the following estimates

$$
u_{+}(t, x)=\bar{u}(t) \bar{f}(\xi) \geq u(t, x) \geq u_{-}(t, x)=\bar{u}(t) \bar{f}(\xi)
$$

\section{Results of the Numerical Experiments}

Problem (1) - (2) has no analytical solution. Therefore, we will discuss result of the numerical experiments. To find a solution of problem at some point we are using numerical methods (see [29]-[31]). The resulting asymptotic of the solutions were used as an initial approximation for numerical computation.

\subsection{One Dimensional Case $(\mathrm{N}=1)$}

From problem (1) - (2) we have following one dimensional nonlinear heat equation with initial and boundary conditions 


$$
\begin{gathered}
\frac{\partial u}{\partial t}=\frac{\partial}{\partial x_{1}}\left(u^{m-1}\left|\frac{\partial u^{k}}{\partial x}\right|^{p-2} \frac{\partial u}{\partial x}\right)-u^{\beta_{*}} \\
\mathrm{u}(0, \mathrm{x})=\psi(\mathrm{x}) \geq 0,0 \leq \mathrm{x} \leq \mathrm{b} \\
\left\{\begin{array}{l}
\mathrm{u}(\mathrm{t}, 0)=\varphi_{1}(\mathrm{t})>0 \\
\mathrm{u}(\mathrm{t}, \mathrm{b})=\varphi_{2}(\mathrm{t})=0
\end{array}, \mathrm{t} \in[\mathrm{O}, \mathrm{T}]\right.
\end{gathered}
$$

For problem (18) we construct the spatial grid $\mathrm{x}$ with steps $\mathrm{h}$

$$
\bar{\omega}_{\mathrm{h}}=\left\{\mathrm{x}_{\mathrm{i}}=\mathrm{ih}, \quad \mathrm{h}>0, \quad \mathrm{i}=0,1, \ldots, \mathrm{n}, \quad \mathrm{hn}=\mathrm{b}\right\}
$$

And temporary grid with $\tau$

$$
\bar{\omega}_{\tau}=\left\{t_{j}=j \tau, \quad \tau>0, \quad j=0,1, \ldots, m, \quad \tau m=T\right\}
$$

replace problem (18) implicit two-layer difference scheme and obtain the difference task with error $\mathrm{O}\left(\mathrm{h}^{2}+\tau\right)$

$$
\left\{\begin{array}{c}
\frac{y_{i}^{j+1}-y_{i}^{j}}{\tau}=\frac{1}{h^{2}}\left[a_{i+1}\left(y^{j+1}\right)\left(y_{i+1}^{j+1}-y_{i}^{j+1}\right)-a_{i}\left(y^{j+1}\right)\left(y_{i}^{j+1}-y_{i-1}^{j+1}\right)\right] \\
\quad-\left(y_{i}^{j+1}\right)^{\beta}, i=1,2, \ldots, n-1 ; \quad j=0,1, \ldots, m-1 \\
y_{i}^{0}=u_{0}\left(x_{i}\right), \quad i=0,1, \ldots, n \\
y_{0}^{j}=\phi_{1}\left(t_{j}\right), \quad j=1,2, \ldots, m \\
y_{n}^{j}=\phi_{2}\left(t_{j}\right), \quad j=1,2, \ldots, m
\end{array}\right.
$$

Where $a_{i+1}$ and $a_{i}$ is nonlinear terms of the problem (18). In our case $a_{i+1}$ and $a_{i}$ are a thermal conductivity coefficients. To calculate $a_{i+1}$ and $a_{i}$, use the following formulas [29]

$$
\begin{aligned}
& a_{i+1}\left(y^{j+1}\right)=\frac{1}{2}\left[\begin{array}{l}
\left(y_{i}^{j+1}\right)^{m-1}\left|\frac{\left(y_{i+1}^{j+1}\right)^{k}-\left(y_{i}^{j+1}\right)^{k}}{h}\right|^{p-2}+ \\
+\left(y_{i+1}^{j+1}\right)^{m-1}\left|\frac{\left(y_{i}^{j+1}\right)^{k}-\left(y_{i-1}^{j+1}\right)^{k}}{h}\right|^{p-2}
\end{array}\right] \\
& a_{i}\left(y^{j+1}\right)=\frac{1}{2}\left[\begin{array}{l}
\left(y_{i-1}^{j+1}\right)^{m-1}\left|\frac{\left(y_{i}^{j+1}\right)^{k}-\left(y_{i-1}^{j+1}\right)^{k}}{h}\right|^{p-2}+ \\
+\left(y_{i}^{j+1}\right)^{m-1}\left|\frac{\left(y_{i-1}^{j+1}\right)^{k}-\left(y_{i-2}^{j+1}\right)^{k}}{h}\right|^{p-2}
\end{array}\right]
\end{aligned}
$$

when $\mathrm{i}=1 \quad a_{i}$ goes beyond the points, so following Milne formulas can be used[31]

$$
\begin{gathered}
\left.\frac{d u}{d x}\right|_{0} \approx \frac{-y_{2}+4 y_{1}-3 y_{0}}{2 h} \text { or } \\
\left.\frac{d u}{d x}\right|_{n} \approx \frac{3 y_{n}-4 y_{n-1}+y_{n-2}}{2 h}
\end{gathered}
$$

Scheme (19) is absolutely stable and has the first order of approximation for $\tau$, and the second for $h$ [29].

System of algebraic equation (19) is nonlinear is relative $\mathrm{y}^{\mathrm{j}+1}$

For solve a system of nonlinear equations (19), we apply an iterative method and obtain following system of algebraic equation [29]

$$
\begin{array}{r}
\frac{y_{i}^{j+1}-y_{i}^{j}}{\tau}=\frac{1}{h^{2}}\left[a_{i+1}\left(y^{s+1}\right)\left(\begin{array}{c}
s+1 \\
y_{i+1}^{j+1}-y_{i}^{j+1}
\end{array}\right)\right. \\
\left.-a_{i}\left(\stackrel{s}{s+1}^{j+1}\right)\left(\begin{array}{c}
s+1 \\
y_{i}^{j+1}-y_{i-1}^{s+1}
\end{array}\right)\right]-\left(y_{i}^{j+1}\right)^{\beta}
\end{array}
$$

where $s=0,1,2, \ldots$ initial and boundary conditions unchanged

Now system of algebraic equation (3.1.5) is linear is relative $\mathrm{y}^{\mathrm{j}+1}$. As the initial iteration for $\stackrel{\mathrm{s}+1}{\mathrm{y}_{\mathrm{i}}} \mathrm{y}$ is taken from the previous time step $\mathrm{y}^{\mathrm{j}+1}=\mathrm{y}^{\mathrm{j}}$. When counting by an iterative scheme, the accuracy $\varepsilon$ of the iteration is set and the process continues until execution the following conditions

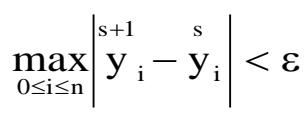

Remark. In all numerical calculations we take $\varepsilon=10^{-3}$

Let following notation

$$
y^{j}=y, y^{j+1}=\bar{y} .
$$

From different scheme (22) we will find tridiagonal matrix coefficients A, B, C, F and solve following system linear equations by method Thomas [29]

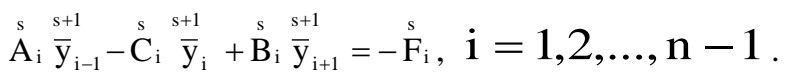

$$
\begin{aligned}
& y_{0}=\chi_{1} y_{1}+\mu_{1} \\
& y_{N}=\chi_{2} y_{N-1}+\mu_{2}
\end{aligned}
$$

Where

$$
\begin{gathered}
\stackrel{s}{A_{i}}=\frac{\tau a_{i}\left(a^{j+1}\right)}{h^{2}}, \stackrel{s}{B_{i}}=\frac{\tau a_{i+1}^{s}\left(a^{j+1}\right)}{h^{2}}, \\
\stackrel{\mathrm{s}}{\mathrm{C}}_{\mathrm{i}}=\stackrel{\mathrm{s}}{\mathrm{A}}_{\mathrm{i}}+\stackrel{\mathrm{s}}{\mathrm{B}}_{\mathrm{i}}+1, \stackrel{s}{F_{i}}=y_{i}-\left(y_{i}^{j+1}\right)^{\beta}, \\
\mathrm{s}=0,1,2 \ldots, \mathrm{i}=1,2, \ldots, \mathrm{n} .
\end{gathered}
$$

\subsection{Two-Dimensional Case $(\mathrm{N}=2)$}

From problem (1) - (2) we have following two-dimensional nonlinear heat equation with initial and boundary conditions 


$$
\begin{gathered}
\frac{\partial u}{\partial t}=\left(\begin{array}{c}
\frac{\partial}{\partial x_{1}}\left(u^{m-1}\left|\frac{\partial u^{k}}{\partial x_{1}}\right|^{p-2} \frac{\partial u}{\partial x_{1}}\right)+ \\
+\frac{\partial}{\partial x_{2}}\left(u^{m-1}\left|\frac{\partial u^{k}}{\partial x_{2}}\right|^{p-2} \frac{\partial u}{\partial x_{2}}\right)
\end{array}\right)-u^{\beta_{*}} \\
\left.u\right|_{\Gamma}=\mu(x, t), \mathrm{u}(0, \mathrm{x})=\mathrm{u}_{0}(\mathrm{x}) \geq 0, \\
0 \leq \mathrm{x}_{\alpha} \leq \mathrm{b}_{\alpha}, \quad \alpha=1,2 .
\end{gathered}
$$

We construct the spatial grid $\mathrm{x}_{\alpha}(\alpha=1,2)$ with steps $\mathrm{h}_{1}=\frac{\mathrm{b}_{1}}{\mathrm{n}_{1}}$ and $\mathrm{h}_{2}=\frac{\mathrm{b}_{2}}{\mathrm{n}_{2}}$ :

$\bar{\omega}_{h}=\left\{x_{i j}=\left(x_{1}^{i}, x_{2}^{j}\right), \quad x_{1}^{i}=i h_{1}, \quad x_{2}^{j}=j h_{2}, \quad i, j=0,1, \ldots, n_{\alpha}, \quad \alpha=1,2\right\}$

And time grid with $\tau$

$$
\begin{gathered}
\bar{\omega}_{\tau}=\left\{t_{k}=l \tau, \quad \tau>0, \quad l=0,1, \ldots, m, \quad \tau m=T\right\}, \\
\mathrm{T}>0 .
\end{gathered}
$$

For numerical solution of the Problem (23) used method of variable direction [30]

$$
\left\{\begin{array}{c}
\frac{y_{i, j}^{l+1 / 2}-y_{i, j}^{l}}{0.5 \cdot \tau}=\Lambda_{1} y^{l+1 / 2}+\Lambda_{2} y^{l}+q\left(y^{l}\right) \\
\frac{y_{i, j}^{l+1}-y_{i, j}^{l+1 / 2}}{0.5 \cdot \tau}=\Lambda_{1} y^{l+1 / 2}+\Lambda_{2} y^{l+1}+q\left(y^{l+1}\right)
\end{array}\right.
$$

where

$$
\begin{gathered}
\Lambda_{1} y^{l+1 / 2}=\frac{1}{h_{1}^{2}}\left[a_{i+1, j}\left(y^{l+1 / 2}\right)\left(y_{i+1, j}^{l+1 / 2}-y_{i, j}^{l+1 / 2}\right)-a_{i, j}\left(y^{l+1 / 2}\right)\left(y_{i, j}^{l+1 / 2}-y_{i-1, j}^{l+1 / 2}\right)\right], \\
\Lambda_{2} y^{l}=\frac{1}{h_{2}^{2}}\left[b_{i, j+1}\left(y^{l}\right)\left(y_{i, j+1}^{l}-y_{i, j}^{l}\right)-b_{i, j}\left(y^{l}\right)\left(y_{i, j}^{l}-y_{i, j-1}^{l}\right)\right], \\
\Lambda_{2} y^{l+1}=\frac{1}{h_{2}^{2}}\left[b_{i, j+1}\left(y^{l+1}\right)\left(y_{i, j+1}^{l+1}-y_{i, j}^{l+1}\right)-b_{i, j}\left(y^{l+1}\right)\left(y_{i, j}^{l+1}-y_{i, j-1}^{l+1}\right)\right] \\
\mathrm{i}, \mathrm{j}=1,2, \ldots, \mathrm{n}_{\alpha}-1, \quad \alpha=1,2 . \\
q(y)=-u^{\beta}
\end{gathered}
$$

For nonlinear part $a_{i, j}\left(y^{l}\right)$ and $a_{i, j}\left(y^{l}\right)$ were used the following approximations

$$
a_{i+1, j}\left(y^{l+1 / 2}\right)=\frac{1}{2}\left[\begin{array}{l}
\left(y_{i, j}^{l+1 / 2}\right)^{m-1}\left|\frac{\left(y_{i+1, j}^{l+1 / 2}\right)^{k}-\left(y_{i, j}^{l+1 / 2}\right)^{k}}{h_{1}}\right|^{p-2}+ \\
+\left(y_{i+1, j}^{l+1 / 2}\right)^{m-1}\left|\frac{\left(y_{i, j}^{l+1 / 2}\right)^{k}-\left(y_{i-1, j}^{l+1 / 2}\right)^{k}}{h_{1}}\right|^{p-2}
\end{array}\right]
$$

$a_{i, j}\left(y^{l+1 / 2}\right)=\frac{1}{2}\left[\begin{array}{l}\left(y_{i-1, j}^{l+1 / 2}\right)^{m-1}\left|\frac{\left(y_{i, j}^{l+1 / 2}\right)^{k}-\left(y_{i-1, j}^{l+1 / 2}\right)^{k}}{h_{1}}\right|^{p-2}+ \\ +\left(y_{i, j}^{l+1 / 2}\right)^{m-1}\left|\frac{\left(y_{i-1, j}^{l+1 / 2}\right)^{k}-\left(y_{i-2, j}^{l+1 / 2}\right)^{k}}{h_{1}}\right|^{p-2}\end{array}\right]$

$b_{i, j+1}\left(y^{l}\right)=\frac{1}{2}\left[\begin{array}{l}\left(y_{i, j}^{l}\right)^{m-1}\left|\frac{\left(y_{i, j+1}^{l}\right)^{k}-\left(y_{i, j}^{l}\right)^{k}}{h_{2}}\right|^{p-2}+ \\ +\left(y_{i+1, j}^{l}\right)^{m-1}\left|\frac{\left(y_{i, j}^{l}\right)^{k}-\left(y_{i-1, j}^{l}\right)^{k}}{h_{2}}\right|^{p-2}\end{array}\right]$

$b_{i, j}\left(y^{l}\right)=\frac{1}{2}\left[\begin{array}{l}\left(y_{i, j-1}^{l}\right)^{m-1}\left|\frac{\left(y_{i, j}^{l}\right)^{k}-\left(y_{i, j-1}^{l}\right)^{k}}{h_{2}}\right|^{p-2}+ \\ +\left(y_{i, j}^{l}\right)^{m-1}\left|\frac{\left(y_{i, j-1}^{l}\right)^{k}-\left(y_{i, j-2}^{l}\right)^{k}}{h_{2}}\right|^{p-2}\end{array}\right]$

$b_{i, j+1}\left(y^{l+1}\right)=\frac{1}{2}\left[\begin{array}{l}\left(y_{i, j}^{l+1}\right)^{m-1}\left|\frac{\left(y_{i, j+1}^{l+1}\right)^{k}-\left(y_{i, j}^{l+1}\right)^{k}}{h_{2}}\right|^{p-2}+ \\ +\left(y_{i+1, j}^{l+1}\right)^{m-1}\left|\frac{\left(y_{i, j}^{l+1}\right)^{k}-\left(y_{i-1, j}^{l+1}\right)^{k}}{h_{2}}\right|^{p-2}\end{array}\right]$

$b_{i, j}\left(y^{l+1}\right)=\frac{1}{2}\left[\begin{array}{l}\left(y_{i, j-1}^{l+1}\right)^{m-1}\left|\frac{\left(y_{i, j}^{l+1}\right)^{k}-\left(y_{i, j-1}^{l+1}\right)^{k}}{h_{2}}\right|^{p-2}+ \\ +\left(y_{i, j}^{l+1}\right)^{m-1}\left|\frac{\left(y_{i, j-1}^{l+1}\right)^{k}-\left(y_{i, j-2}^{l+1}\right)^{k}}{h_{2}}\right|^{p-2}\end{array}\right]$

For computation on the end of the segment the Milne formulas (21) is also used.

Scheme (24) is stable both according to the initial data and on the right-hand side for any $\tau$ and $h$. Scheme has accuracy $O\left(\tau^{2}+h^{2}\right)$ [30].

In this scheme, the transition from layer $l$ to layer $l+1$ is carried out in two stages. In first stage find intermediate values for $1+1 / 2$ and in second stages find values for $1+1$ with usages founded value $l+1 / 2$ layer.

Rewrite the initial and boundary conditions as follows 


$$
\left\{\begin{array}{l}
y_{i, j}^{0}=u_{0}(x), \quad x \in \bar{\omega}_{h} \\
y_{i, j}^{l+1}=\phi^{l+1}, \quad \text { when } j=0 \text { и } j=n_{2} \\
y_{i, j}^{l+1 / 2}=\bar{\phi}^{l+1 / 2}, \quad \text { when } \mathrm{i}=0 \text { и } i=n_{1}
\end{array}\right.
$$

where $\bar{\phi}=\frac{1}{2}\left(\phi^{l+1}+\phi^{l}\right)-\frac{\tau}{4} \Lambda_{2}\left(\phi^{l+1}-\phi^{l}\right)$ [30].

Let following notation

$$
y^{l}=y, y^{l+1 / 2}=\bar{y}, y^{l+1}=\hat{y} .
$$

For solve a system of nonlinear equations (3.2.2), we apply an iterative method and obtain following system of algebraic equation

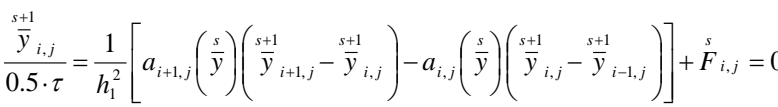

$$
\begin{aligned}
& \frac{\stackrel{s}{y}_{i, j}}{0.5 \cdot \tau}=\frac{1}{h_{2}^{2}}\left[b_{i, j+1}\left(\begin{array}{l}
s \\
\hat{y}
\end{array}\right)\left(\begin{array}{cc}
s+1 & s+1 \\
\hat{y}_{i, j+1} & -\hat{y}_{i, j}
\end{array}\right)-b_{i, j}\left(\begin{array}{c}
s \\
\hat{y}
\end{array}\right)\left(\begin{array}{cc}
s+1 & s+1 \\
\hat{y}_{i, j} & -\hat{y}_{i, j-1}
\end{array}\right)\right]+\stackrel{s}{F}_{i, j}=0
\end{aligned}
$$

Now system of algebraic equation (26) is linear to relative $\stackrel{s+1}{\mathrm{y}}_{i, j},(27)$ is linear is relative $\stackrel{\mathrm{s}}{\hat{\mathrm{y}}}_{\mathrm{i}, \mathrm{j}}$. As the initial iteration in (26) for $\stackrel{s+1}{\mathrm{y}}_{\mathrm{i}, \mathrm{j}} \mathrm{y}$ is taken from the previous time step $\overline{\mathrm{y}}_{\mathrm{i}, \mathrm{j}}=\mathrm{y}_{\mathrm{i}, \mathrm{j}}$. When counting by an iterative scheme, the accuracy $\varepsilon$ of the iteration is set and the process continues until execution the following conditions

$$
\max _{\substack{0 \leq i \leq n_{1} \\ 0 \leq j \leq n_{2}}}\left|\frac{s+1}{\bar{y}_{i, j}}-\bar{y}_{i, j}\right|<\varepsilon
$$

As the initial iteration in (3.2.5) for $\hat{\mathrm{y}}_{\mathrm{i}, \mathrm{j}}^{\mathrm{s+1}} \mathrm{y}$ is taken from the previous time step $\hat{\mathrm{y}}_{\mathrm{i}, \mathrm{j}}=\overline{\mathrm{y}}_{\mathrm{i}, \mathrm{j}}$. When counting by an iterative scheme, the accuracy $\varepsilon$ of the iteration is set and the process continues until execution the following conditions

$$
\max _{\substack{0 \leq i \leq \mathrm{n}_{1} \\ 0 \leq \mathrm{j} \mathrm{n}_{2}}}\left|\hat{\mathrm{y}}_{\mathrm{i}, \mathrm{j}}^{\mathrm{s}+1}-\hat{\mathrm{y}}_{\mathrm{i}, \mathrm{j}}\right|<\varepsilon
$$

Remark. In all numerical calculations we take $\varepsilon=10^{-3}$

Difference equation (26) can be written as

$$
\left\{\begin{array}{l}
\stackrel{s}{A}_{i, j} \stackrel{s+1}{y}_{i+1, j}-\stackrel{s}{C}_{i, j} \stackrel{s+1}{y}_{i, j}+\stackrel{s}{B}_{i, j} \stackrel{s+1}{y}_{i-1, j}=-\stackrel{s}{F}_{i, j} \\
\bar{y}=\phi, \quad \text { when } i=0, n_{1}
\end{array}\right.
$$

$$
\mathrm{i}, \mathrm{j}=1,2, \ldots, \mathrm{n}_{\alpha}-1, \quad \alpha=1,2
$$

and (27) as

$$
\left\{\begin{array}{l}
\bar{A}_{i, j} \hat{y}_{i, j+1}^{s+1}-\bar{C}_{i, j} \hat{y}_{i, j}^{s+1}+\bar{B}_{i, j} \hat{y}_{i, j-1}^{s+1}=-\bar{F}_{i, j} \\
\hat{y}=\bar{\phi}, \quad \text { when } j=0, n_{2}
\end{array}\right.
$$

After we have 3 diagonal matrix equations with following coefficients A, B, C, F and can solve following system linear equations by method Thomas.

$$
\begin{aligned}
& \stackrel{s}{A_{i}}=\frac{\tau a_{i, j}^{s}\left(\bar{y}^{l+1 / 2}\right)}{2 h_{1}^{2}}, \quad \stackrel{s}{B}{ }_{i}=\frac{\tau a_{i+1, j}^{s}\left(\bar{y}^{l+1 / 2}\right)}{2 h_{1}^{2}}, \\
& \stackrel{\mathrm{s}}{\mathrm{C}}_{\mathrm{i}}=\stackrel{\mathrm{s}}{\mathrm{A}_{\mathrm{i}}}+{\stackrel{\mathrm{s}}{\mathrm{B}_{\mathrm{i}}}+1}^{\prime}, \stackrel{s}{F}_{i}=\bar{y}_{i, j}^{l}-\frac{\tau}{2}\left(\bar{y}_{i, j}^{l}\right)^{\beta}+\frac{\tau}{2} \Lambda_{2} \vec{s}^{l}, \\
& \bar{A}_{i}=\frac{\tau b_{i, j}^{s}\left(\hat{y}^{l+1}\right)}{2 h_{2}^{2}} \quad \bar{s}_{i}=\frac{\tau a_{i, j+1}^{s}\left(\hat{y}^{l+1}\right)}{2 h_{2}^{2}}, \\
& \overline{\mathrm{C}}_{i}=\overline{\mathrm{A}}_{i}+\overline{\mathrm{B}}_{i}+1 \text {, } \\
& \frac{s}{F}_{i}=\hat{y}_{i, j}^{l+1 / 2}-\frac{\tau}{2}\left(\hat{y}_{i, j}^{l+1 / 2}\right)^{\beta}+\frac{\tau}{2} \Lambda_{1} \hat{y}^{l+1 / 2}
\end{aligned}
$$

The system of equations (28) is solved by lines $\mathrm{j}=1,2, \ldots, \mathrm{n}_{2}-1$ and $\overline{\mathbf{y}}$ determined at all point of grid $\omega_{\mathrm{h}}$, then system of equations (29) is solved by columns $\mathbf{i}=1,2, \ldots, \mathrm{n}_{1}-1$ and $\widehat{\mathbf{y}}$ determined at all point of grid $\omega_{\mathrm{h}}$. During the transition from layer $\mathrm{l}+1$ to layer $1+2$, the counting procedure is repeated.

\subsection{Visualization}

Notice that very important to found appropriate initial approximation of solution depending on value of numerical parameters. Therefore, an initial and a boundary values are calculated using the founded above following asymptotic

$$
u_{0}(t, x)=(t \ln t)^{-\frac{1}{\beta_{*}-1}} \bar{f}(\xi), \beta_{*}=k(p-2)+m+\frac{p}{N}
$$

$$
\bar{f}(\xi)=\left(a-b \xi^{\frac{p}{p-1}}\right)_{+}^{\frac{p-1}{m+k(p-2)-1}}
$$

$$
\begin{aligned}
& \xi=x[\tau(t)]^{-1 / p}, \tau(t)=t^{\alpha_{1}} \ln ^{\alpha_{2}} t \\
& \alpha_{1}=\frac{k(p-2)+m-1}{(p+(k(p-2)+m) N) p}, \\
& \alpha_{2}=-\frac{k(p-2)+m-1}{(p+(k(p-2)+m) N) p}+1
\end{aligned}
$$$$
b=(m+k(p-2)-1) /\left(k^{p-2}\right)\left(p^{p}\right)^{\frac{1}{p-1}}, m+k(p-2)-1>0,
$$ 


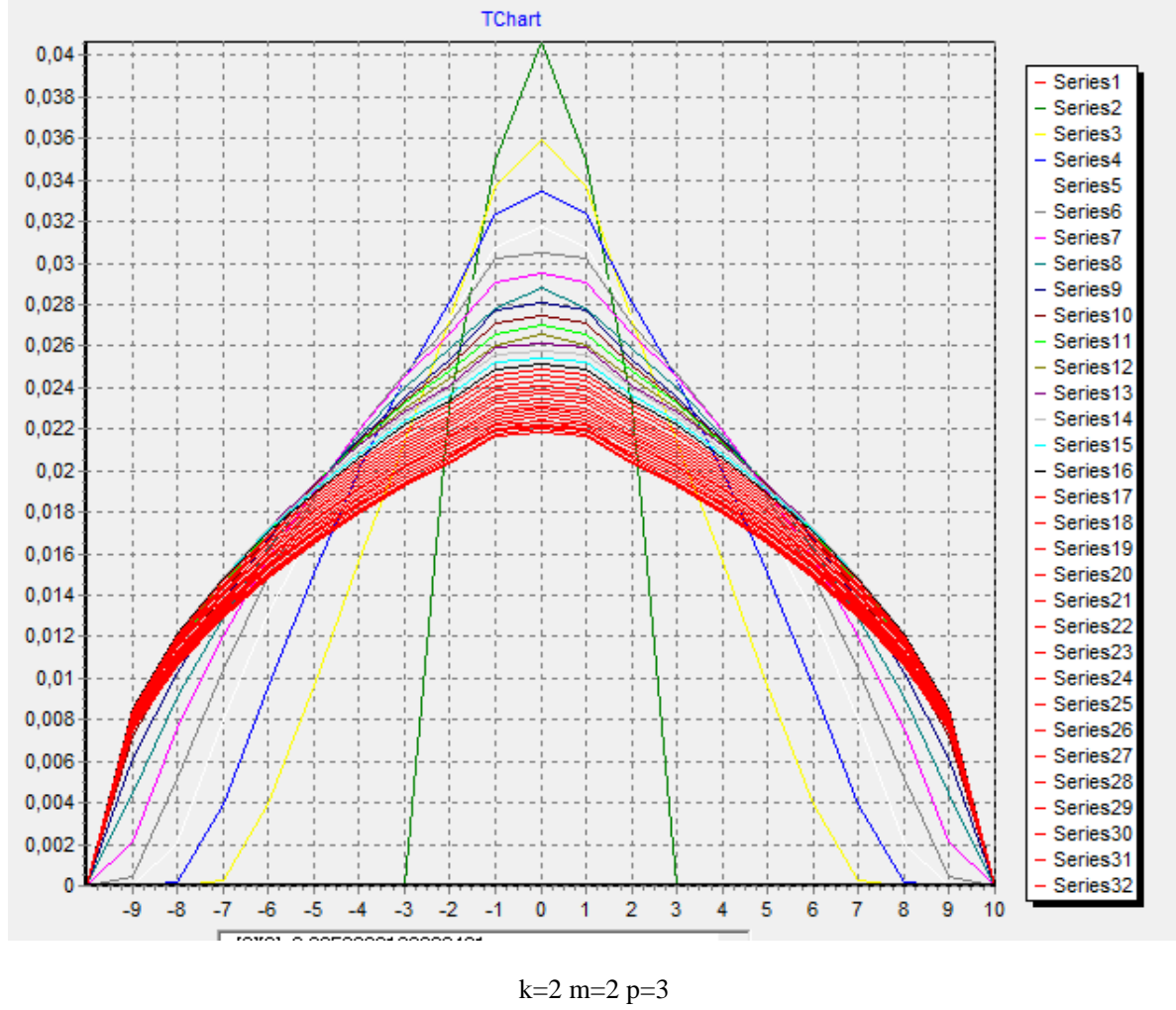

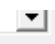

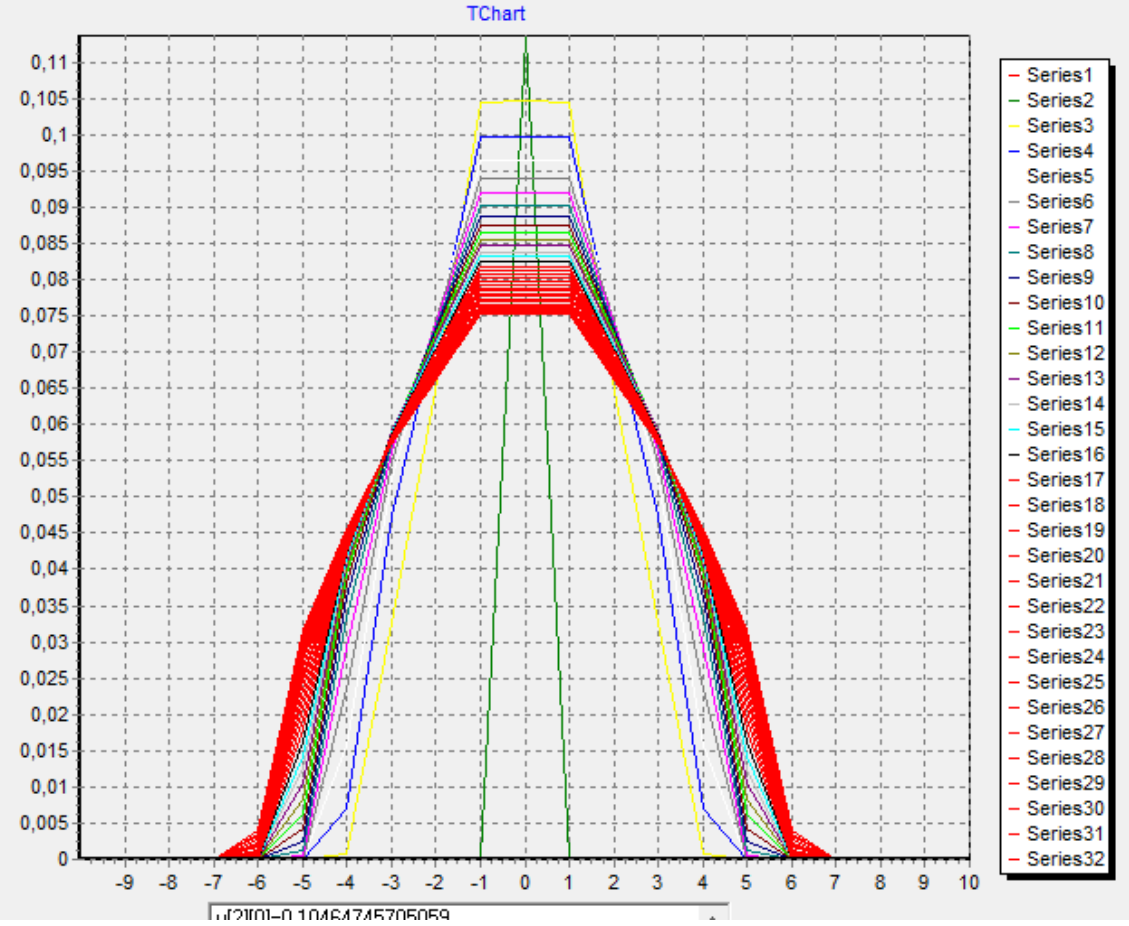

$p=4 m=4 k=2$ 


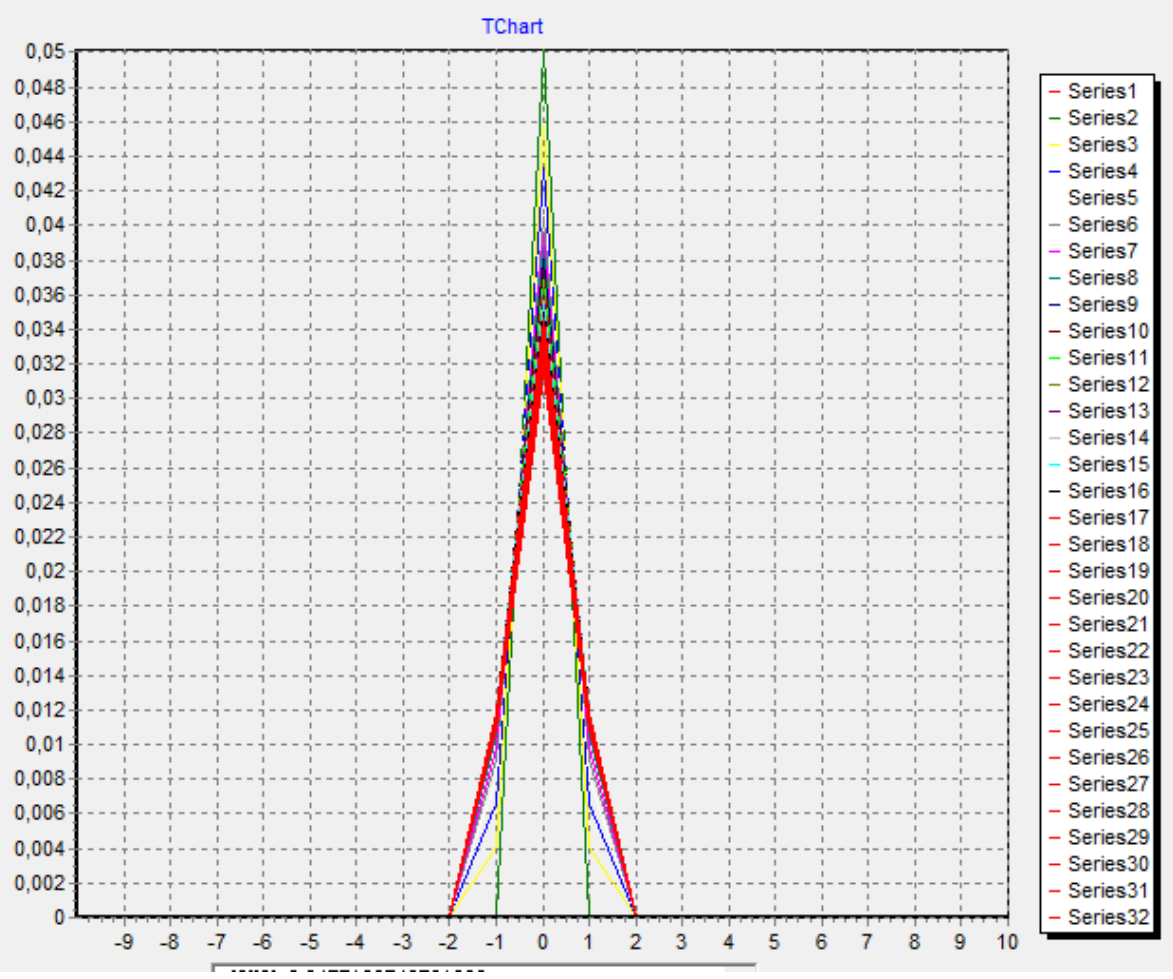

$$
\mathrm{m}=4 \mathrm{p}=3 \mathrm{k}=2
$$

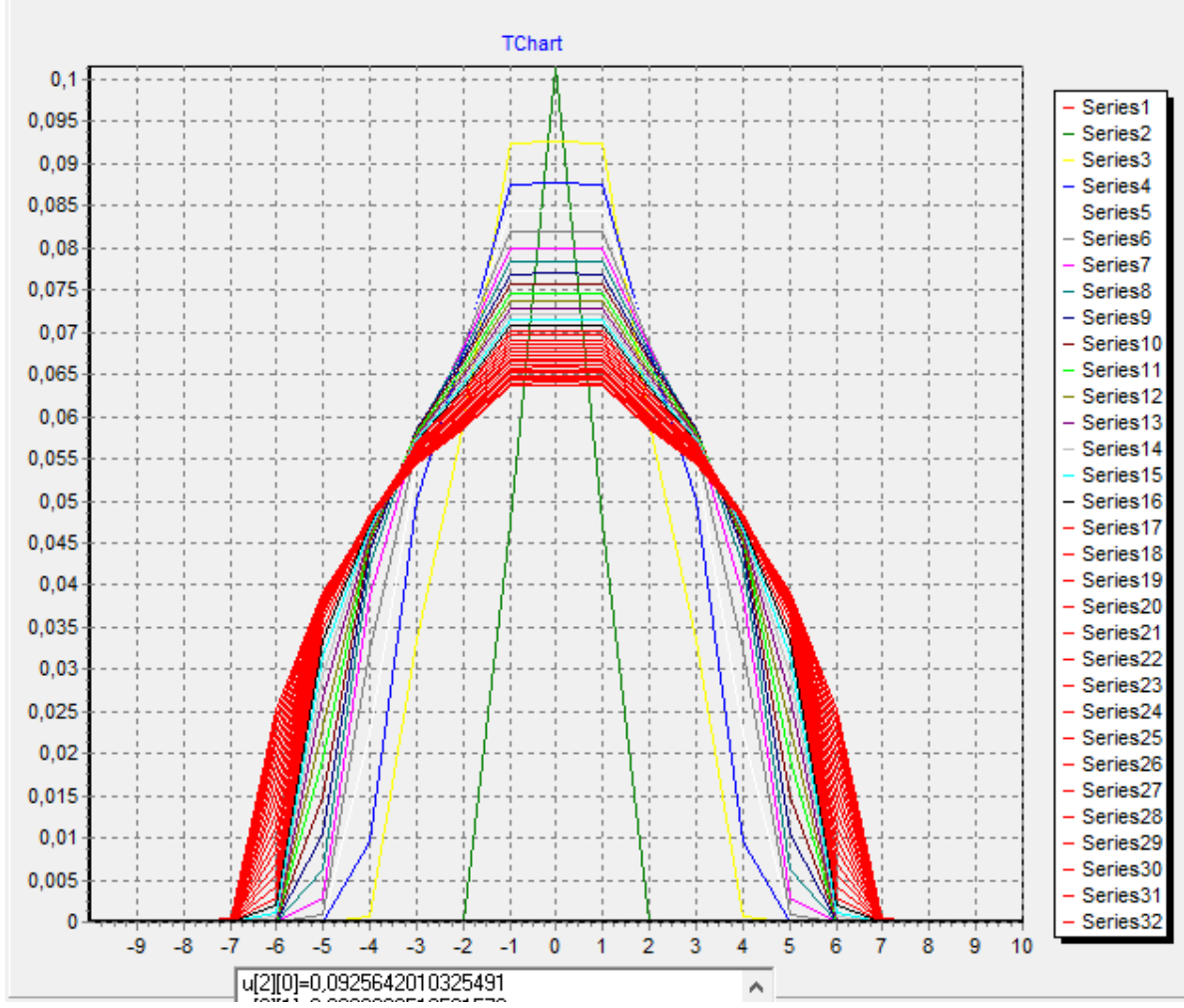

$$
\begin{aligned}
& k=4 p=3 m=2 \\
& k=2 m=2 p=3
\end{aligned}
$$




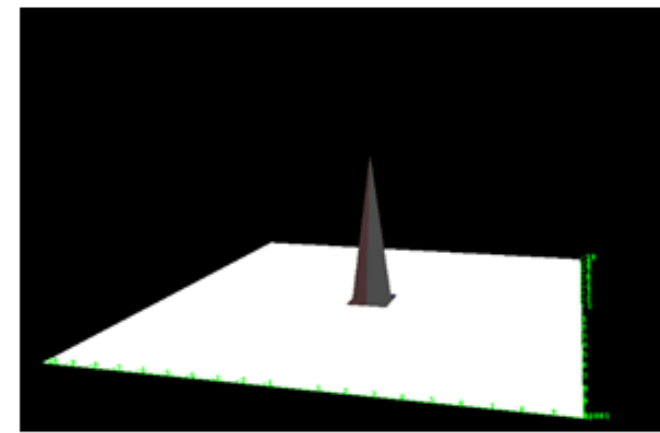

$t=1$

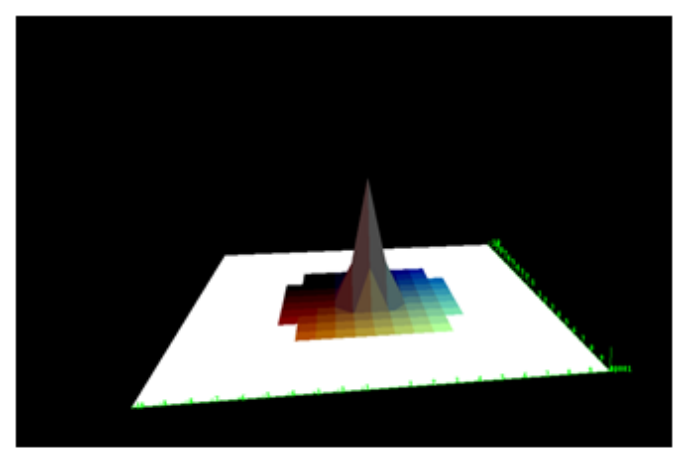

$t=10$

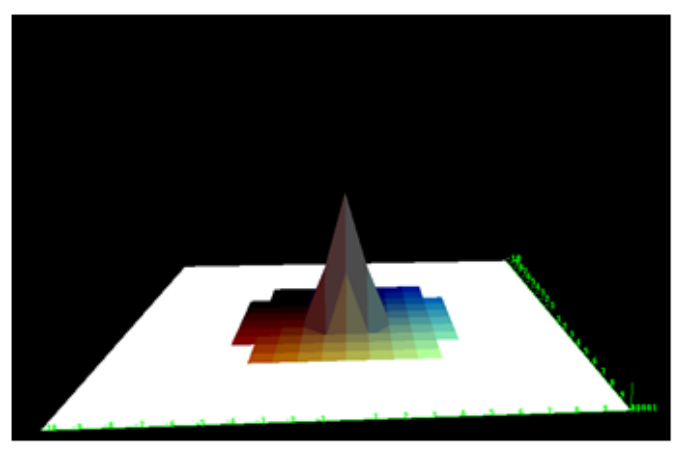

$\mathbf{t}=\mathbf{2 0}$

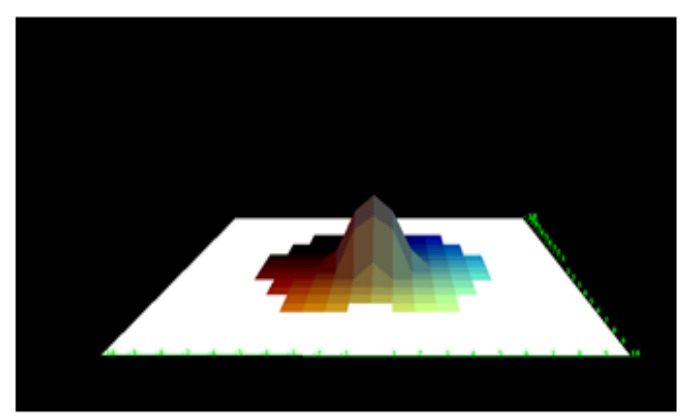

$t=30$

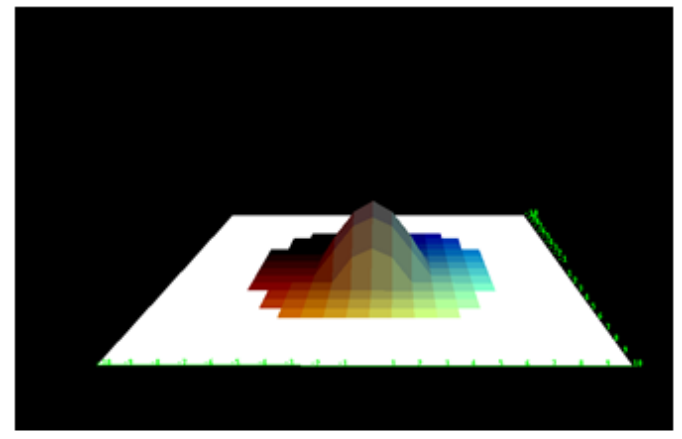

$t=50$

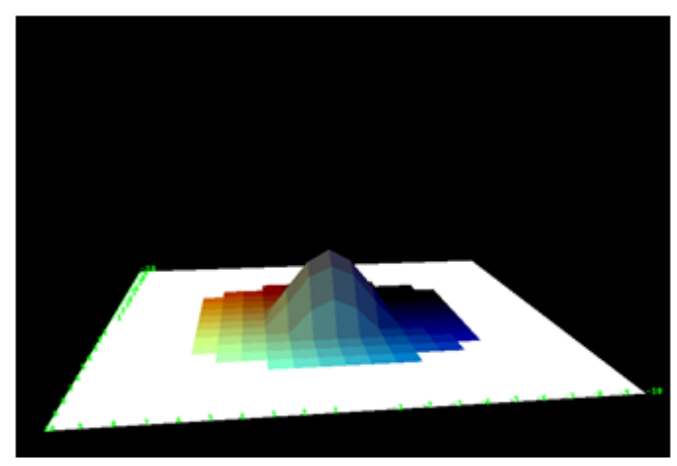

$t>100$

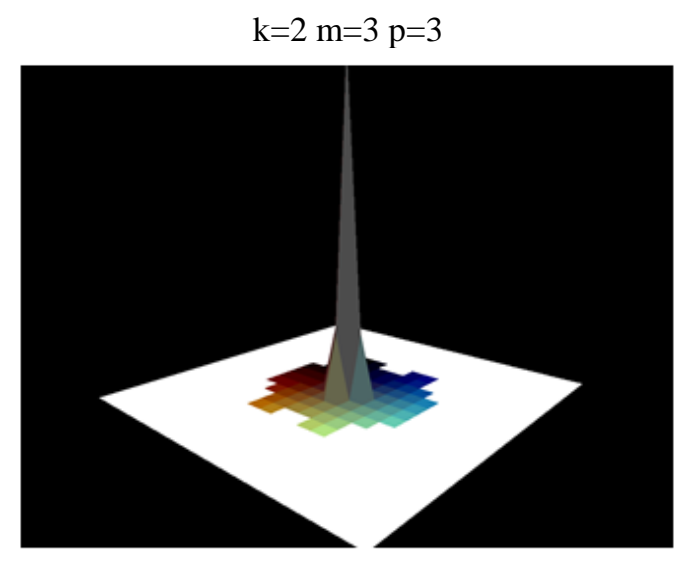

$\mathbf{t}=\mathbf{1 0}$

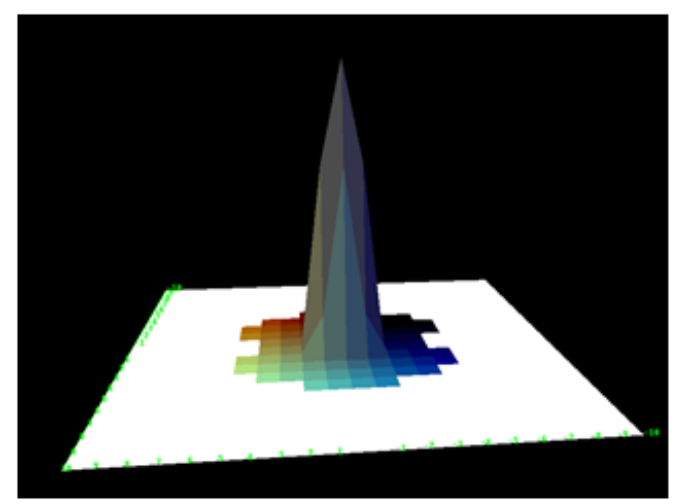

$t=30$ 


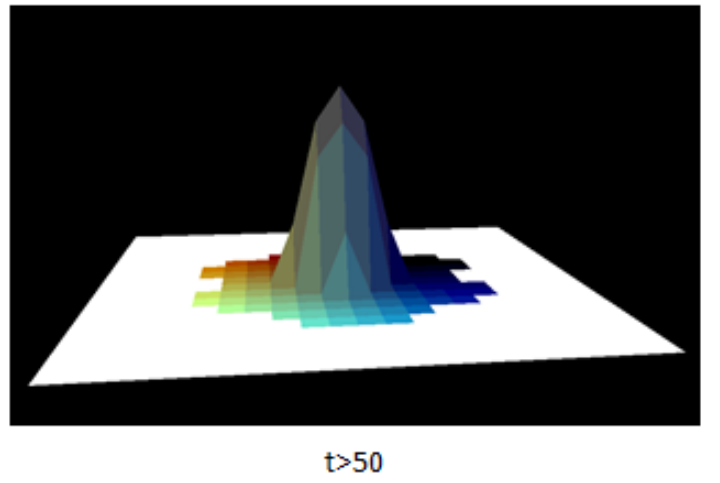

$\mathrm{k}=1 \mathrm{~m}=2 \mathrm{p}=3$

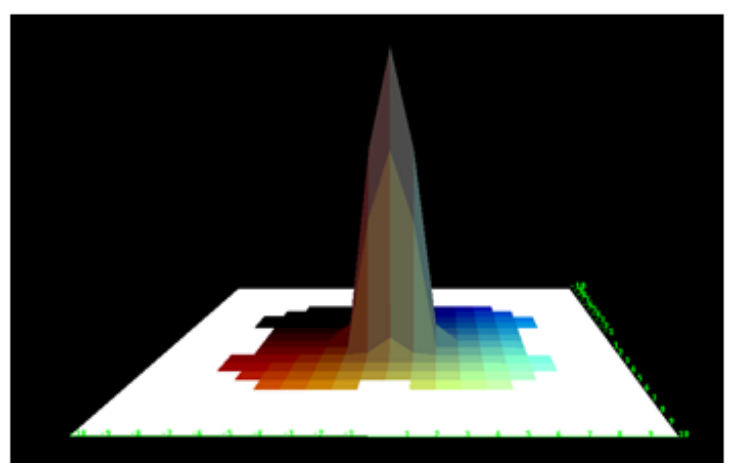

$t=5$

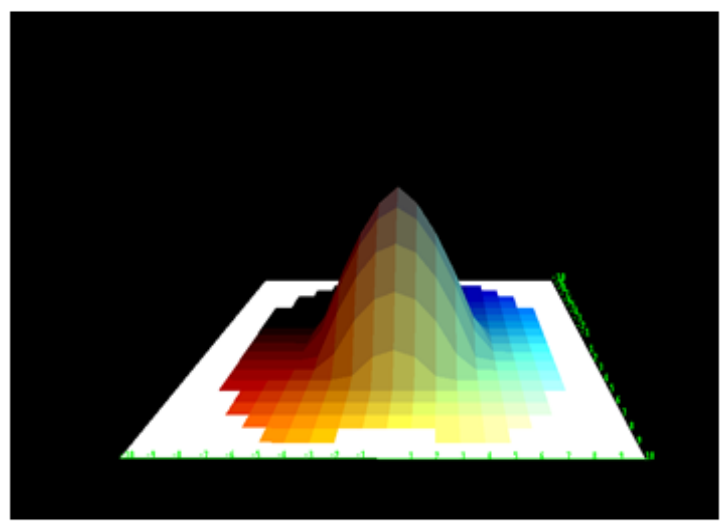

$t=20$

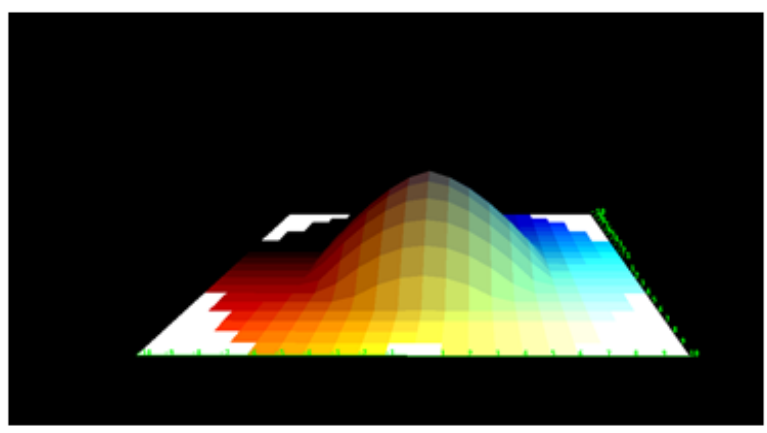

$t>50$

As we can see from the figures, for large times and a critical value of parameter $\beta_{*}$, the solutions of the problem infinitely close to each other, which leads to localization.

\section{Conclusions}

As we see the asymptotic of the solution to problem (1)-(2) for the critical parameter $\beta$ changes its asymptotic for $t \rightarrow+\infty$. Proofs of the proposal based on principle of comparison solutions. The resulting asymptotic of the solutions were used as an initial approximation for numerical computation. As we now the final propagation velocity is a natural effect inherent in nonlinear processes. In all numerical experiments we got this effect. As a numerical experiment showed, founded asymptotic is a good initial approximation. The numerical results are in good agreement with the physics of this process.

We are currently investigating the convergence of schemes (24), (19) and the asymptotic stability of a solution (30).

In[32] authors study the homogeneous Dirichlet problem for the following doubly nonlinear equation posed in a bounded domain in $R^{N}$ with homogeneous boundary conditions and with non-negative and integrable data for the degenerate case $m(p-1)>1$ and the quasilinear case $m(p-1)=1$

$$
\begin{cases}u_{t}(t, x)=\Delta_{p} u^{m}(t, x), & \text { for } t>0 \text { and } x \in \Omega, \\ u(0, x)=u_{0}(x), & \text { for } x \in \Omega, \\ u(t, x)=0, & \text { for } t>0 \text { and } x \in \partial \Omega .\end{cases}
$$

$\Delta_{p} w:=\operatorname{div}\left(|\nabla w|^{p-2} \nabla w\right)$ is $\mathrm{p}$-Laplacian operator, $\mathrm{p}>1$, $\mathrm{m}>0$.

They established the following long time asymptotic of the solution and gives rates

$$
u(t, x)=(t+s)^{-\alpha} h(r), \quad r=|x|(t+s)^{-\beta},
$$

where $\alpha, \beta$ are positive parameters and $h$ a real valued function satisfying a certain ODE.

The founded long time asymptotic of the solution (3.3.1) can be used for this problem with the additional absorption part for the critical parameter $\beta$ in case $\mathrm{m}=1, \mathrm{k}=\mathrm{m}$.

In addition, the equation (17) can be obtained by multiplying the equation (15) on $\xi^{N-1}$ and integrating over the interval $(0, \xi)$. Kind of such an equation may be get using work [33].

At $k(p-2)+m=1$ there is a special case. In this case function $\bar{f}(\xi)$ looks like following

$$
\bar{f}(\xi)=\exp \left(-\left(k^{p-2} p^{p}\right)^{\frac{1}{p-1}} \xi^{\frac{p}{p-1}}\right)
$$

All this suggest that parameter $\beta$ is very sensitive to parameters $\mathrm{k}, \mathrm{p}, \mathrm{m}$. Besides at $k(p-2)+m=1$ the asymptotic of the solution will also change, which as in the 
critical case, can discover new effects, properties, estimates.

For the equation with variable density which is given below, the critical value of parameter $\beta$ is also found. With it helps search the asymptotic of the solution with critical parameter possible.

$$
\begin{gathered}
|x|^{-l} \frac{\partial u}{\partial t}=\nabla\left(u^{m-1}\left|\nabla u^{k}\right|^{p-2} \nabla u\right)-|x|^{-l} u^{\beta_{*}}, \\
u(0, x)=u_{0}(x), x \in R^{N} \\
u(t, x)=w(t, \varphi(x)) \\
\frac{\partial w}{\partial t}=\varphi^{1-s} \frac{\partial}{\partial \varphi}\left(\varphi^{s-1} w^{m-1}\left|\frac{\partial w^{k}}{\partial \varphi}\right|^{p-2} \frac{\partial w}{\partial \varphi}\right)-w^{\beta_{*}} \\
\varphi(|x|)=p|x|^{(p-l)} /(p-l), s=p \frac{N-l}{p-l}, N>l, l<p \\
\beta_{*}=k(p-2)+p / s=k(p-2)+\frac{p-l}{N-l}
\end{gathered}
$$

The search for new effects of a variable density problem for a critical value of parameter $\beta$ is a very important and interesting study. It expands the possibilities of applied application of this study and this equation a more generalized case of problem (1) - (2).

\section{REFERENCES}

[1] H. Fujita, "On the blowing up of solutions of the Cauchy problem for $u_{t}=\Delta u+u^{1+\alpha}$ ”, University of Tokyo, Journal of the Faculty of Science A, vol. 16, pp. 105-113, 1966.

[2] M.Aripov, Method of standard equation for solving nonlinear boundary value problems, Fan, Tashkent, 1986.

[3] P. Cianci, A. V. Martynenko, and A. F. Tedeev, "The blow-up phenomenon for degenerate parabolic equations with variable coefficients and nonlinear source,” Nonlinear Analysis: Theory, Methods \& Applications A, vol. 73, no. 7, pp. 2310-2323, 2010.

[4] E. Di Benedetto, Degenerate Parabolic Equations, Universitext, Springer, New York, NY, USA, 1993.

[5] J. N. Zhao, “On the Cauchy problem and initial traces for the evolution p-Laplacian equations with strongly nonlinear sources,” Journal of Differential Equations, vol. 121, no. 2, pp. 329-383, 1995.

[6] Z. Wu, J. Zhao, J. Yun and F. Li, Nonlinear Diffusion Equations, Singapore: World Scientific Publishing, New York, 2001.

[7] A. Gmira, On quasilinear parabolic equations involving measure date, North-Holland, Asymptotic Analysis, vol 3, pp. 43-56.1990.

[8] J. Yang and J. Zhao, A note to the evolutional P-Laplace equation with absorption, Acta. Sci. Nat. Jilin., Vol 2, pp. 35-38, 1995.

[9] J. Zhao, Source-type solutions of quasilinear degenerate parabolic equation with absorption, Chin. Ann. of Math., ISB1, pp. 89-104, 1994.

[10] J. Zhao, Existence and nonexistence of solution for $u_{t}=\operatorname{div}\left(|\nabla u|^{p-2} \nabla u\right)+f(\nabla u, u, x, t)$, J. Math. Anal. Appl. Vol. 172, pp. 130-146, 1993.

[11] J. Zhao, The Cauchy problem for $u_{t}=\operatorname{div}\left(|\nabla u|^{p-2} \nabla u\right)$ when $2 \mathrm{~N} /(\mathrm{N}+1)<\mathrm{p}<2$, T.M.A., Nonlinear Anal. vol. 24, pp. 615-630, 1995.

[12] J. Zhao and H. Yuan, The Cauchy problem of a class of doubly degenerate parabolic equation (in chinese), Chinese Ann. Of Math. 16As2, pp. 181-196, 1995.

[13] E. Dibenedetto and A. Friedman, HOlder estimates for nonlinear degenerate parabolic systems, J. reine. Angew. Math. Vol.357, pp. 1- 22, 1985.

[14] Y. Li and Ch. Xie, Blow-up for p-Laplace parabolic equations, E. J. D. E., vol. 20, pp. 1-12, 2003.

[15] E. Dibenedetto and M. A.Herrero, On Cauchy problem and initial traces for a degenerate parabolic equations, Trans.Amer. Soc. Vol.314, pp. 187-224, 1989.

[16] Ph. Benilan, M. G. Crandall and M. Pierre, Solutions of the porous medium equation in $\mathrm{RN}$ under optimal conditions on initial values, Indiana Univ., Math. J. vol. 33, pp. 51-71, 1984.

[17] J. Zhao and Z. Xu, Cauchy problem and initial traces for a doubly degenerate parabolic equation, Sci.in China, Ser.A, vol. 39, pp. 673-684, 1996.

[18] H. Fan, Cauchy problem of some doubly degenerate parabolic equations with initial datum a measure, Acta Math. Sinica, EnglishSer. Vol 20, pp. 663-682, 2004.

[19] J. L. V'azquez and V. A. Galaktionov, Asymptotic behavior of solutions of the nonlinear absorption diffusion equation at a critical exponent,report of the Academy of Sciences of USSR, N, p, 1989.

[20] V. A. Galaktionov and S. A. Posashkov, Asymptotics of nonlinear heat conduction with absorption under the critical exponent, Keldysh Inst. Appl. Math.,Preprint no. 71, Acad. Sci. USSR,. P, (1986).

[21] V.A.Galaktionov, S.P. Kurdyumov, A.A. Samararskiy, $\{$ On asymptotic "eigenfunctions" of the Cauchy problem for a nonlinear parabolic equation, mathematical collection, Volume 126 (168), Number 4, p. 435-472, 1985.

[22] V. A. Galaktionov and J. L. V'azquez, Asymptotic behaviour of nonlinear parabolic equations with critical exponents. A dynamical systems approach, J. Funct. Anal., vol.100, 435-462, 1991.

[23] Andreucci D., Tedeev A.F. Universal bounds at the blow-up time for nonlinear parabolic equations. Adv. Differential Equations vol.10, no. 1, 89-120, 2005.

[24] Andreucci D., Tedeev A.F., Ughi M. The Cauchy problem for degenerate parabolic equations with source and damping. Ukr. Mat. Visn. Vol.1, no. 1, pp. 1-19, 2004. 
[25] Victor A. Galaktionov Critical Global Asymptotics In Higher-Order Semilinear Parabolic Equations, Hindawi Publishing Corp. Ijmms, vol.60, pp.3809-3825, 2003.

[26] C. Mu, Y. Li, and Y. Wang, "Life span and a new critical exponent for a quasilinear degenerate parabolic equation with slow decay initial values,” Nonlinear Analysis, vol. 11, no. 1, pp. 198-206, 2010.

[27] J.-S. Guo and Y. Y. Guo, "On a fast diffusion equation with source,” The Tohoku Mathematical Journal, vol. 53, no. 4, pp. 571-579, 2001.

[28] Said Benachour, Razvan Iagar, Philippe Laurencot. Large time behavior for the fast diffusion equation with critical absorption. Journal of Differential Equations, Elsevier, vol.260, pp.8000-8024, 2016.

[29] A.A. Samararskiy, A.V. Gulin, "numerical methods", “Nauka”, p.p 432, 1989.

[30] A.A. Samararskiy, introduction to the theory of difference schemes, “Nauka”, p.p 553, 1971.

[31] Aripov M., "Applied mathematics in science and technology”,’Tashkent”, p.p186, 2008

[32] Diana Stan and Juan Luis Vazquez Asymptotic behavior of the doubly nonlinear equation $u_{t}=\Delta_{p} u^{m}$ on bounded domainsar, Elseiver, Nonlinear analysis, vol.77, pp.1-32, 2013.

[33] Aripov M., Mukimov A. “An asymptotic solution radially symmetric self-similar solution of nonlinear parabolic equation with source in the second critical exponent case ", National university of Uzbekistan, ACTA NUUz, vol.2, n.2, p.21-30, 2017. 